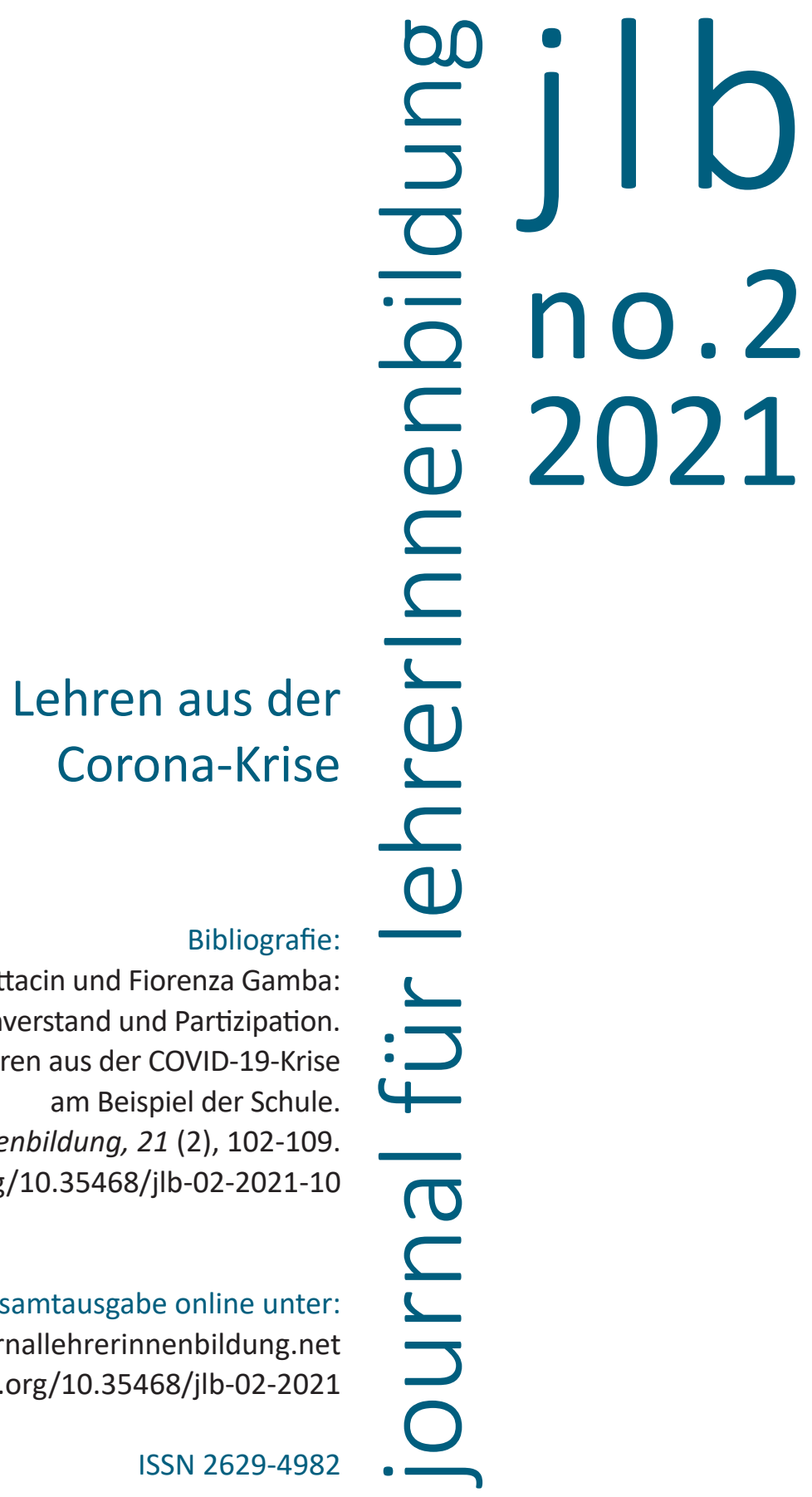


10

Sandro Cattacin und Fiorenza Gamba

\section{Sachverstand und Partizipation. Lehren aus der COVID-19-Krise am Beispiel der Schule}


Wissen ist Macht und Macht muss kontrolliert werden, sollen grundlegende Freiheiten garantiert bleiben. Wäre dies eine Gleichung, wür-

de sie etwa so aussehen: $M^{a}=W+D$, wobei Macht $(M)$ längerfristig nur aufgeklärt $\left(M^{\mathrm{a}}\right)$ - das heißt vernünftig - sein kann, wenn diese demokratisch (D) kontrolliert wird und auf Wissen (W) aufbaut, ohne von diesem beherrscht zu werden. Bevor wir weitere Leser*innen verlieren, möchten wir kurz ankünden, worum es in diesem Text geht. Wir möchten am Beispiel der Schule zu COVID-19-Zeiten aufzeigen, wie Wissen, Macht und Demokratie zusammenspielen.

\section{Unwissendes Wissen}

Wissen ist grundsätzlich kontextabhängiges Unwissen, das mit einem unbekannten Ablaufdatum versehen ist. Bis weit ins Mittelalter herrschte die Idee vor, die Erde sei flach. Die Theorie der runden Erde setzte sich nur sehr langsam durch. Der allgemeine Wissensstand verändert sich somit ständig. So spiegelten auch die spärlich zusammengetragenen Informationen über die Pandemie den unwissenden Sachverstand: Helfen Masken oder Schließungen überhaupt, welche Vakzine wirken besser und sollen Menschen aus dem Impfangebot auswählen dürfen? Nur schon die unterschiedlichen Debatten in der Wissenschaft weisen darauf hin, dass in dieser Krise vor allem politische Antworten nötig sind, was auch in Bezug auf Schulschließungen gilt. Wissenschaft ist zwar argumentativ aufgebaut, doch rekonstruktiv ausgerichtet. Daher wird die Wissenschaft erst in ein paar Jahren (vielleicht) einen mehrheitsfähigen Konsens erarbeitet haben, der es erlaubt, die Pandemie besser zu verstehen. Wissenschaft braucht also Zeit, aber nicht nur.

Wissensproduktion geht zwar oft schrittweise vor - vorangetrieben durch die Falsifizierbarkeit von Hypothesen, wie Karl Popper (1959) sagen würde -, doch wird diese manchmal auch völlig auf den Kopf gestellt. Thomas Kuhn (1957) spricht dann von einem Paradigmenwechsel. Wir können also nicht vollständig auf den Sachverstand vertrauen, doch diesem oft gut gemeinten Wissen auch nicht nur mit Misstrauen begegnen.

Auch in der Coronakrise ist (leider) ein Paradigmenstreit im Gang. Dabei geht es um die Frage, welche Gesellschaft wir uns wünschen. Eine Gesellschaft, in der jede*r Tote zu vermeiden ist, in der überlebende 
Körper wichtiger sind als deren Lebensqualität? Diese durchaus provokative Frage beschäftigt Wissenschaft und Gesellschaft schon seit einiger Zeit. Wobei die körperorientierte, nach Foucault „biopolitische" Ausrichtung (Foucault, 2004) sich eher auf dem Rückzug zu befinden schien. Selbstbestimmung im Sterben und Sterben in Würde, Schadensminderung, Fürsorge statt „Reparatur", sind beispielhafte Stichworte für das, was eine andere Art des Messens gesellschaftlichen Fortschritts sein könnte (Stiglitz, Sen \& Fitoussi, 2010).

Biomedizinische Sichtweisen stoßen auf soziale (und ökonomische ${ }^{1}$ ) Sichtweisen und beschäftigen uns inzwischen tagtäglich, wobei das Resultat des Aufeinandertreffens dieser Sichtweisen meist konservativ ausfällt und auf dem eigentlich überholten biomedizinischen Modell basiert. Doch ungeachtet dessen mischen sich, quasi als Korrektiv, zivilgesellschaftliche Organisationen und innovative Dienste im Sozialbereich ein und gewichten die Pflege von Menschen und Beziehungen höher als das individuelle Überleben.

Die Schulen verkörpern in diesem paradigmatischen Konflikt gleichzeitig engagierte Pionierinnen, mühsame Spielverderberinnen und eigentliche Nebenwelten. Seit Beginn der Pandemie ist die SchulschlieBung für die einen Pflicht, für die anderen Unfug. Das Resultat ist ein Hin und Her, eine pädagogische Herausforderung, was erneut beweist, wie wichtig Flexibilität im Erziehungssystem ist. Doch scheint das Pendel im Paradigmenstreit eher für die Zukunft auszuschlagen: Schulen sind primär nicht dazu da, die Verbreitung der Krankheit zu bremsen, sondern sind zentrale Orte im Kampf für eine gerechtere Gesellschaft. Das Beispiel England ist dabei besonders hervorzuheben, das selbst in der größten Krisensituation im Januar 2021, Schulen für vulnerable Kinder und Kinder mit Eltern, die nicht zu Hause bleiben können, offen hält (BBC, 2021).

\section{Wissen und Macht}

Wissensproduktion hat also meist hehre Motive, aber nicht immer. Michel Foucault hat sich in seinen ersten Studien darauf versteift,

1 Wobei die ökonomische Sichtweise - anders als die soziale - vor allem auf die fehlende Berechenbarkeit der Zukunft als Basis wirtschaftlichen Handelns sowie die Verantwortung des privaten Unternehmertums in Krisenzeiten hinwies (Crane \& Matten, 2020). 
uns den Zusammenhang zwischen Machtmissbrauch und Wissen aufzuzeigen. Macht legitimiere sich durch Wissen und Wissen schütze Mächtige, um zu überleben (Foucault, 1969). Es überrascht deshalb kaum, dass autoritäre Systeme die Wissensproduktion überwachen (Gómez, Balmer \& Canales, 2015), technokratische Systeme Demokratien aushöhlen (Habermas, 1968) und die Politik gerne Wissen und Forschung instrumentalisiert (Lukes, 1974). Dabei sind insbesondere Sozialwissenschaften im Fokus der Kontrolle und Beeinflussung. ${ }^{2}$ Deren unnahbarste, nahezu platonische Variante erzählt uns verstörende Geschichten, denen wir zuhören können oder eben nicht, wobei deren unangenehmste, nämlich die sokratische Variante nicht aufhört, uns Fragen zu stellen, deren Nicht-Beantwortung zu noch hartnäckigerem Nachfragen führt. Und dies alles mit der aufklärerischen Hoffnung, dass beantwortete Fragen uns einen Schritt weiterbringen.

Die COVID-19-Krise ist ein gutes Beispiel dieser vermeintlich produktiven Verbindung von Wissen und Macht. China - unbestritten ein autoritäres System - brachte zuerst Alarmierende zum Schweigen und sperrte danach "modellhaft“ Menschen in ganzen Städten ein - basierend auf wissenschaftlichen Erkenntnissen. Doch unsere Demokratien glänzten auch nicht gerade. In Italien wurden kritische Berichte zur veralteten Pandemiestrategie verheimlicht, in den Vereinigten Staaten die Wissenschaft der Lächerlichkeit ausgesetzt, in der Schweiz (und nicht nur) Seminare unter Epidemiolog*innen im Fernsehen durchgeführt (was zwar zur Illustration der Logik der Wissenschaften lehrreich war, aber fatal für deren Glaubwürdigkeit; siehe dazu Gamba, Cattacin, Ricciardi \& Nardone, 2020).

\section{Partizipation}

Das beste bisher bekannte Gegenmittel zu Machtmissbrauch ist die Kombination von demokratischer Öffentlichkeit, Partizipation und Gewaltenteilung, alles in sich sehr anfällige Elemente, doch zusammen eine ziemlich starke Arznei, die darauf ausgerichtet ist, respektvollen Umgang zwischen Personen herzustellen. Bei dieser Kontrolle ist auch

2 Aber nicht nur, sondern seit jeher auch die Erforschung der gesundheitlichen Konsequenzen des Tabakkonsums, der Atomenergie oder der Klimaerwärmung. Beispielhaft dazu die Studie von Chung-Yol Lee und Stanton Glantz (2001). 
Wissen relevant, das die demokratische Entscheidungsfindung speist. Dieser Herausforderung sind sich Wissensproduzierende, die sich seit Jahren dafür einsetzen, dieses Wissen allen zugänglich zu machen, sehr wohl bewusst. Open Source und Open Data Archives sind digitale Antworten darauf, partizipative Forschung steht für die entsprechende methodologische Ausrichtung (Nielsen, 2011).

Die politische Antwort auf COVID-19 ist bisher kein lehrreiches Beispiel demokratischen Prozederes. Top-down-Entscheide müssen systematisch durch Bottom-up-Bewegungen korrigiert werden. Zivilgesellschaftliche Organisationen haben alle Hände voll zu tun, um den durch die Politik Vergessenen Würde zukommen zu lassen. Sans-papiers, psychisch oder kognitiv Beeinträchtigte jeglichen Alters, nationale Grenzen überschreitende Beziehungen aller Art - und alle diesen nahestehenden Personen - stehen hier als Beispiele für eine solche politische Amnesie vor allem der ersten Stunde.

Auch Schüler*innen wurden kaum gefragt, was denn für sie eine gute Lösung wäre (Stoecklin, 2020). Die Kraft demokratischer Schulung zeigte sich während dieser Krise besonders deutlich, als das Lehrpersonal eigene Taktiken einsetzte, um weiterhin unterrichten zu können. Doch trotz vieler heroischer Lehrender scheiterten Schulschließungen erfolgreich. Erfolgreich daran war, dass die Politik sich in vielen Ländern einsetzte, den Fernunterricht für alle zu ermöglichen. Doch letztlich ist Fernunterricht an den vielen Ansprüchen gescheitert, die wir mit Schule, Ausbildung, persönlicher Entwicklung, Gerechtigkeit, Elternsein und vielem mehr verbinden.

Wenn Politik, Schule und Elternhaus scheitern, sind Kinder zwar machtlos, doch Jugendliche und Lehrende können sich wehren. In Mailand besetzen Gymnasiast*innen beispielsweise ihre Schule, tragen Maske, bestehen auf Distanz und gute Lüftung und organisieren Schnelltests, damit Schule wieder möglich wird. Lehrende in Norwegen organisieren „Draußenschulen“ und Professor*innen in Genf machen Spaziergänge mit Studierenden.

\section{Demokratische Wissensgesellschaften}

Wissensgesellschaften sind offene Gesellschaften, die die einfache und generelle Teilnahme an Wirtschaft, Politik und Wissenschaft als aufklärerisches Projekt der Moderne hochhalten. Schulen und Lehren- 
de spielen dabei eine zentrale Rolle, denn sie sind mitverantwortlich, vielleicht sogar hauptverantwortlich, für die Bildung autonomer Subjekte, die sich demokratische Regeln aneignen. Dies um sich selbst, aber auch unsere Gesellschaft vor technokratischer oder autoritärer Gewalt zu schützen und diese über gewaltlose Konfrontation voranzubringen (Rawls, 1971). Die COVID-19-Krise hat uns allen vorgeführt, worum es uns im Grunde geht: In Würde leben und überleben. Das biomedizinische Modell war zwar politisch lange die Hauptorientierung, konnte dies aber nur dank des Engagements aller anderen Akteuren - zivilgesellschaftliche Organisationen, Schulen, einzelne Bürger*innen - bleiben, so dass dessen Rezepte wenigstens halbwegs wirkten. Das nach Jahren des Einsatzes für eine gesunde Schule gerade diese zum Tummelplatz unüberlegter Politik und nicht zum Zentrum innovativer Lösungen wurde, sollte uns nachdenklich stimmen. Wo denn sonst als in der Welt des Lernens soll Innovation stattfinden? Die COVID-19-Pandemie zeigt uns deshalb eindrücklich auf, dass Schulen nicht verkrusten dürfen, und nicht nur an morgen, sondern bereits an übermorgen denken sollten. Macht muss in diesem Sinne (auch kritische) Räume der Innovation öffnen, um gesellschaftliche Probleme konstruktiv und partizipativ zu lösen. Und die Wissensproduktion darf dabei nicht vergessen, dass diese nur dann frei sein kann, wenn Macht demokratischer Kontrolle unterstellt ist. Eben: $M=W+D$.

Sandro Cattacin und Fiorenza Gamba sind Forschende am Institut der recherches sociologiques der Universität Genf. Zusammen haben sie daran mitgewirkt, das erste Buch zu COVID-19 aus sozialwissenschaftlicher Perspektive zu veröffentlichen (Gamba, F., Ricciardi, T., Nardone, M. \& Cattacin, S. (Hrsg.). (2020). COVID-19. Eine sozialwissenschaftliche Perspektive. Zürich u. a.: Seismo).

\section{Literatur}

BBC (2021). Covid: What is happening with schools? BBC News (06012021). Verfügbar unter https://www.bbc.com/news/education-51643556 [13.04.2021].

Crane, A. \& Matten, D. (2020). COVID-19 and the Future of CSR Research. Journal of Management Studies, 50. https://doi.org/10.1111/joms.12642.

Foucault, M. (1969). L'archéologie du savoir. Paris: Gallimard.

Foucault, M. (2004). Naissance de la biopolitique: cours au Collège de France (19781979). Paris: Gallimard. 
Gamba, F., Cattacin, S., Ricciardi, T. \& Nardone, M. (2020). Sozial- und Humanwissenschaften als Orientierungswissenschaften. In F. Gamba, M. Nardone, R. Ricciardi \& S. Cattacin (Hrsg.), COVID-19. Eine sozialwissenschaftliche Perspektive (S. 343358). Genf u. a.: Seismo.

Gómez, A., Balmer, B. \& Canales, A. F. (2015). Science policy under democracy and dictatorship: An introductory essay. In A. Gómez, A. F. Canales \& B. Balmer (eds.), Science Policies and Twentieth-Century Dictatorships: Spain, Italy and Argentina (pp. 1-26). London a. o.: Routledge.

Habermas, J. (1968). Technik und Wissenschaft als Ideologie. Frankfurt am Main: Suhrkamp.

Kuhn, T. S. (1957). The Copernican revolution; planetary astronomy in the development of Western thought. Cambridge: Harvard University Press.

Lee, C.-Y. \& Glantz, S. A. (2001). The tobacco industry's successful efforts to control tobacco policy making in Switzerland. San Francisco: University of California, Institute for Health Policy Studies.

Lukes, S. (1974). Power. A Radical View. London: MacMillan.

Nielsen, M. (2011). Reinventing discovery: the new era of networked science. Princeton: University Press.

Popper, K. R. (1959). The logic of scientific discovery. New York: Basic Books.

Rawls, J. (1971). A Theory of Justice. Mass.: Harvard University Press.

Stiglitz, J. E., Sen. A \& Fitoussi, J.-P. (2010). Mismeasuring Our Lives. New York: The New Press.

Stoecklin, D. (2020). Kinder und die COVID19-Krise. In F. Gamba, M. Nardone, R. Ricciardi \& S. Cattacin (Hrsg.), COVID-19. Eine sozialwissenschaftliche Perspektive (S. 205-227). 
Sandro Cattacin, Prof. Dr., Direktor des Instituts de recherches sociologiques an der Universität Genf. Arbeitsschwerpunkte: Stadtsoziologie, Lebensstile und Marginalität, translokale Mobilität

Sandro.Cattacin@unige.ch

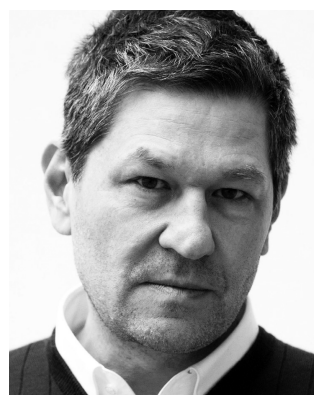

Fiorenza Gamba, Prof. Dr. Dr., Institut de recherches sociologiques an der Universität Genf, Professorin an der Universität Sassari. Arbeitsschwerpunkte: Digitale Medien, Lebensstile, Stadtanthropologie

Fiorenza.Gamba@unige.ch

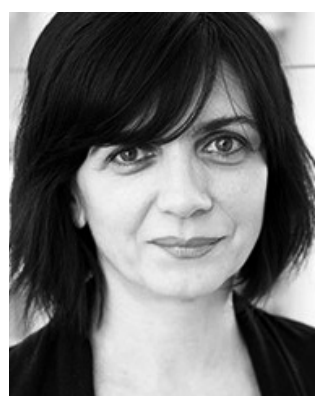

